\title{
Leishmania amazonensis exhibits phosphatidylserine-dependent procoagulant activity, a process that is counteracted by sandfly saliva
}

\author{
Natalia Cadaxo Rochael', Luize Gonçalves Lima², \\ Sandra Maria Pereira de Oliveira ${ }^{3}$, Marcello André Barcinski ${ }^{4}$, \\ Elvira Maria Saraiva', Robson Queiroz Monteiro ${ }^{2}$, Lucia Helena Pinto-da-Silva ${ }^{5}{ }^{+}$ \\ 'Departamento de Imunologia, Instituto de Microbiologia Paulo de Góes ²Instituto de Bioquímica Médica, \\ Universidade Federal do Rio de Janeiro, Rio de Janeiro, RJ, Brasil ${ }^{3}$ Departamento de Entomologia \\ ${ }^{4}$ Laboratório de Biologia Celular, Instituto Oswaldo Cruz-Fiocruz, Rio de Janeiro, RJ, Brasil \\ ${ }^{5}$ Instituto de Veterinária, Universidade Federal Rural do Rio de Janeiro, Seropédica, RJ, Brasil
}

Leishmania parasites expose phosphatidylserine (PS) on their surface, a process that has been associated with regulation of host's immune responses. In this study we demonstrate that PS exposure by metacyclic promastigotes of Leishmania amazonensis favours blood coagulation. L. amazonensis accelerates in vitro coagulation of human plasma. In addition, L. amazonensis supports the assembly of the prothrombinase complex, thus promoting thrombin formation. This process was reversed by annexin $V$ which blocks PS binding sites. During blood meal, Lutzomyia longipalpis sandfly inject saliva in the bite site, which has a series of pharmacologically active compounds that inhibit blood coagulation. Since saliva and parasites are co-injected in the host during natural transmission, we evaluated the anticoagulant properties of sandfly saliva in counteracting the procoagulant activity of $\mathrm{L}$. amazonensis. Lu. longipalpis saliva reverses plasma clotting promoted by promastigotes. It also inhibits thrombin formation by the prothrombinase complex assembled either in phosphatidylcholine (PC)/PS vesicles or in L. amazonensis. Sandfly saliva inhibits factor X activation by the intrinsic tenase complex assembled on PC/PS vesicles and blocks factor Xa catalytic activity. Altogether our results show that metacyclic promastigotes of $\mathrm{L}$. amazonensis are procoagulant due to PS exposure. Notably, this effect is efficiently counteracted by sandfly saliva.

Key words: sandfly saliva - Leishmania amazonensis - phosphatidylserine - blood coagulation - Lutzomyia longipalpis

Leishmaniasis is a parasitic disease caused by protozoan parasites from the genus Leishmania. Infected host may exhibit from destructive skin lesions to an often fatal, if untreated, visceral disease. Infections are acquired via the bite of female sandflies (Diptera, Psychodidae, Phlebotominae) and the genus Lutzomyia is the predominant leishmaniasis vector in South America. During a blood meal, sandfly lacerates capillaries forming a haemorrhagic pool upon which it feeds, inoculates saliva and regurgitates Leishmania metacyclic promastigote forms. Sandfly saliva possesses an array of pharmacologically active compounds, which helps to locate blood vessels and to disrupt the host haemostatic process (Ribeiro \& Francischetti 2003, Koh \& Kini 2009). Haemostasis includes several pathways towards overcoming blood loss, including vasoconstriction, platelet aggregation and blood coagulation. Several insect salivary molecules have been identified as responsible to counteract host haemostasis. Salivary anticoagulants seem to target specific proteases or enzymatic complexes of the blood coagulation cascade,

doi: 10.1590/0074-0276108062013002

Financial support: CNPq, Carlos Chagas Filho Foundation/FAPERJ, CAPES

+Corresponding author: 1pintosilva@gmail.com

Received 29 January 2013

Accepted 2 July 2013 thus blocking or delaying clot formation and allowing the blood feeder to complete the meal (Monteiro 2005, Koh \& Kini 2009). A number of anticoagulant proteins have been identified in Lutzomyia longipalpis saliva by employing molecular biology and biochemical approaches: a family of coagulation inhibitors belonging to the C-type lectinlike family (Charlab et al. 1999, Valenzuela et al. 2004) and, more recently, a novel factor Xa specific inhibitor, named lufaxin (Collin et al. 2012).

Apoptotic mimicry has been described in a number of pathological organisms, including virus and trypanosomatids (Wanderley et al. 2006, Laliberte \& Moss 2009, Santos et al. 2011). This phenomenon consists in the surface exposure of phosphatidylserine (PS) by viable pathogenic organisms, without death as the necessary outcome. In the case of Leishmania, both parasite forms, promastigotes and amastigotes, expose PS on their surface, which is an important factor for infection establishment and maintenance, since it contributes for modulating the host's immune response (Wanderley \& Barcinski 2010).

Exposure of PS by activated platelets has been recognised as a crucial step during physiological blood coagulation process (Heemskerk et al. 2002, Lentz 2003). In fact, anionic phospholipids facilitate the assembly and activation of multimolecular enzymatic complexes, responsible for activation of blood clotting zymogens (Mann 1999). In this context, pathological exposure of PS by tumour cells (Kirszberg et al. 2009, Lima et al. 2011) or by Plasmodium falciparum-infected erythrocytes (Francischetti 
et al. 2007), for example, favours blood coagulation and contribute to disease progression.

Considering that in the moment of sandfly bite, both saliva and metacyclic promastigotes are injected on a haemorrhagic pool in the host skin and that both seems to have opposite effects on blood coagulation, we investigated here the influence of parasite PS exposure and $L u$. longipalpis saliva on blood coagulation. Our results show that PS exposure by metacyclic promastigotes results in a procoagulant effect. However, the presence of sandfly saliva counteracts this phenomenon therefore reestablishing the inactivation of the blood coagulation cascade.

\section{MATERIALS AND METHODS}

Lu. longipalpis and salivary gland lysates (SGL) - Lu. longipalpis were obtained from Santarém and Camará colonies, maintained at the Department of Entomology, Oswaldo Cruz Foundation, Rio de Janeiro (RJ), Brazil. Salivary glands of three-five days-old non blood-fed adult female $\mathrm{Lu}$. longipalpis were dissected, transferred to microtubes containing phosphate buffer saline (PBS) and lysed by sonication. The homogenates were centrifuged at $10,000 \mathrm{rpm}$ for $1 \mathrm{~min}$ to remove debris and the supernatants were transferred to new tubes and stored to $-80^{\circ} \mathrm{C}$ until use.

Parasites - Leishmania amazonensis (MHOM/BR/75/ Josefa) was maintained on Schneider Insect's Medium supplemented with $10 \%$ foetal calf serum and $2 \%$ human urine at $26^{\circ} \mathrm{C}$. Metacyclic purification was performed according to Späth and Beverley (2001). Briefly, stationary promastigotes of five-six day cultures were collected, washed and resuspended at $1 \times 10^{8} / \mathrm{mL}$ density in RPMI1640. Parasites $(4 \mathrm{~mL})$ were then overlaid on $4 \mathrm{~mL}$ of Ficoll $(10 \%)$ in PBS and centrifuged at room temperature (RT) for $10 \mathrm{~min}$ at $1,300 \mathrm{~g}$ with the brake off. Metacyclic forms were collected in the 10\% Ficoll layer, washed twice in PBS and resuspended in PBS for assays.

Reagents - Factor Xa was purchased from Calbiochem (San Diego, CA, USA). Human factor Va and factor X were purchased from Haematologic Technologies (Essex Junction, VT, USA). Human factor IXa was purchased from American Diagnostica (Greenwich, CT, USA). Human FVIII (Advate) was purchased from Baxter Healthcare Corporation (Westlake Village, CA, USA) and was activated with human thrombin as previously described (Astermark et al. 1992). Chromogenic substrates for factor Xa (S-2765, N- $\alpha$-benzyloxycarbonylD-Arg-Gly-Arg-p-nitroanilide) and thrombin (S-2238, H-D-phenylalanyl-L-pipecolyl-L-arginine-p-nitroanilinedihydrochloride) were purchased from Diapharma (Westchester, OH, USA). Activated partial thromboplastin time (aPTT) (cephalin plus kaolin) and prothrombin time (PT) (thromboplastin with calcium) reagents were from BioMérieux (RJ, Brazil). L- $\alpha$-phosphatidylcholine (PC) and PS were purchased from Sigma Chemical Co (St Louis, MO, USA). Phospholipid vesicles (PC/PS) composed of $75 \%$ PC, $25 \%$ PS (w/w) were prepared by sonication. Briefly, phospholipids in chloroform were dried with a stream of $\mathrm{N} 2$, resuspended in $50 \mathrm{mM}$ Tris$\mathrm{HCl}, 150 \mathrm{mM} \mathrm{NaCl}, \mathrm{pH} 7.5$ and sonicated for $2 \mathrm{~min}$.
Evaluation of plasma coagulation by recalcification time - Plasma coagulation assays by recalcification time were performed on an Amelung KC4A coagulometer (Labcon, Heppenheim, Germany) using plastic tubes. Human blood samples were collected from healthy donors in $3.8 \%$ trisodium citrate $(9: 1, \mathrm{v} / \mathrm{v})$ and platelet poor plasma was obtained by further centrifugation at $2,000 \mathrm{~g}$ for $10 \mathrm{~min}$. The procoagulant activity of $L$. amazonensis was evaluated by incubation of plasma $(50 \mu \mathrm{L})$ with 50 $\mu \mathrm{L}$ of tris buffered saline containing different concentrations of metacyclic promastigotes. After $1 \mathrm{~min}$ incubation at $37^{\circ} \mathrm{C}$, plasma clotting was initiated by addition of $100 \mu \mathrm{L}$ of $12.5 \mathrm{mM} \mathrm{CaCl}_{2}$ and coagulation time was recorded. The anticoagulant activity of $\mathrm{Lu}$. longipalpis SGL was evaluated by incubating $50 \mu \mathrm{L}$ of PBS containing 1 SGL with plasma $(50 \mu \mathrm{L})$. After $1 \mathrm{~min}$ at $37^{\circ} \mathrm{C}$, plasma clotting was initiated by addition of $100 \mu \mathrm{L}$ of $12.5 \mathrm{mM} \mathrm{CaCl}_{2}$ and coagulation time was recorded. For control, plasma was incubated only with PBS. Assays were also performed upon incubation of SGL (various dilutions) with different concentrations of metacyclic forms of L. amazonensis. Plasma clotting was initiated as described above.

Activated partial aPTT and PT tests - Clotting times of human plasma were recorded on a coagulometer after activation of either the intrinsic or the extrinsic coagulation pathways. Platelet-poor plasma was incubated with $50 \mu \mathrm{L}$ of PBS containing or not $1 \mathrm{SGL}$ and $50 \mu \mathrm{L}$ of aPTT reagent for $2 \mathrm{~min}$ at $37^{\circ} \mathrm{C}$. Plasma clotting was started by addition of $100 \mu \mathrm{L}$ of $12.5 \mathrm{mM} \mathrm{CaCl}$ and coagulation time was recorded. For PT assays, $50 \mu \mathrm{L}$ of platelet-poor plasma were incubated with $50 \mu \mathrm{L}$ of PBS containing or not $1 \mathrm{SGL}$ for $1 \mathrm{~min}$ at $37^{\circ} \mathrm{C}$. Reaction was started by addition of $100 \mu \mathrm{L}$ of PT reagent and the plasma clotting was recorded.

Flow cytometric analysis - PS exposure by metacyclic forms of $L$. amazonensis was evaluated by flow cytometric analysis. Parasites were resuspended in annexin $\mathrm{V}$ binding buffer $(10 \mathrm{mM}$ HEPES, $150 \mathrm{mM} \mathrm{NaCl}, 2.5 \mathrm{mM}$ $\mathrm{CaCl}_{2}, \mathrm{pH}$ 7.2) and further incubated for $15 \mathrm{~min}$ at $\mathrm{RT}$ with annexin V-Alexa-488 (Molecular Probes, Eugene, OR, USA) according to the instructions of the manufacturer. Propidium iodide (PI) was added to the samples at the moment of acquisition $(0.4 \mu \mathrm{g} / \mathrm{mL}$, final concentration). The samples (10,000 events) were acquired on FACSCalibur and analysed on Cell Quest software.

Prothrombin activation assay - Activation of prothrombin by the prothrombinase complex (factor Xa/factor $\mathrm{Va} /$ calcium) was performed in $50 \mathrm{mM}$ HEPES, 100 $\mathrm{mM} \mathrm{NaCl}, 5 \mathrm{mM} \mathrm{CaCl}, 1 \mathrm{mg} / \mathrm{mL}$ bovine serum albumin (BSA), pH 7.5 (HEPES-BSA buffer), using a previously described discontinuous assay (Fernandes et al. 2006). Factor $\mathrm{Xa}(10 \mathrm{pM}$, final concentration) was incubated for 2 min at $37^{\circ} \mathrm{C}$ with factor $\mathrm{Va}(1 \mathrm{nM}$, final concentration) and PC/PS ( $20 \mu \mathrm{M}$, final concentration) in the presence of serially diluted SGL. Reaction was initiated by addition of prothrombin $(0.5 \mu \mathrm{M}$, final concentration) and aliquots of $10 \mu \mathrm{L}$ were transferred into microplate wells containing $40 \mu \mathrm{L}$ of Tris-ethylenediamine tetraacetic acid (EDTA) 
buffer (50 mM Tris-HCl, $150 \mathrm{mM} \mathrm{NaCl}, 20 \mathrm{mM}$ EDTA, $1 \mathrm{mg} / \mathrm{mL}$ polyethyleneglycol $6,000, \mathrm{pH} 7.5$ ). After addition of $50 \mu \mathrm{L}$ of $100 \mu \mathrm{M} \mathrm{S}-2238$ prepared in Tris-EDTA buffer, absorbance at $405 \mathrm{~nm}$ was recorded at $37^{\circ} \mathrm{C}$ for 20 min at $6 \mathrm{~s}$ intervals using a Versamax Microplate Reader (Molecular Devices, Menlo Park, CA, USA) equipped with a microplate mixer and heating system. Controls were performed in the absence of SGL. The ability of metacyclic promastigotes in assembling the prothrombinase complex was evaluated by the incubation of factor $\mathrm{Xa}(10 \mathrm{pM}$, final concentration) and factor $\mathrm{Va}(1 \mathrm{nM}$, final concentration) for $2 \mathrm{~min}$ at $37^{\circ} \mathrm{C}$ with $L$. amazonensis in HEPES-BSA buffer. Reaction was initiated by addition of prothrombin $(0.5 \mu \mathrm{M}$, final concentration) and thrombin formation was evaluated as described. The inhibitory effect of SGL on prothrombinase complex assembled in either PC/PS or L. amazonensis was also evaluated. In this case, SGL (at various concentrations) was incubated with factor Xa, factor Va and either PC/PS or L. amazonensis, before addition of prothrombin.

Factor X activation assay - Activation of factor X to factor Xa by the intrinsic tenase complex (factor IXa/ factor VIIIa/calcium) was performed in HEPES-BSA buffer, using a previously described discontinuous assay (Fernandes et al. 2006). Factor IXa (0.2 nM, final concentration) was incubated for $2 \mathrm{~min}$ at $37^{\circ} \mathrm{C}$ with factor VIIIa (10 IU/mL, final concentration) in the presence of serially diluted SGL. Reaction was initiated by addition of factor X (50 nM, final concentration) and after $5 \mathrm{~min}$ at RT, aliquots of $25 \mu \mathrm{L}$ were transferred into microplate wells containing $25 \mu \mathrm{L}$ of Tris-EDTA. After addition of $50 \mu \mathrm{L}$ of $200 \mu \mathrm{M} \mathrm{S}-2765$ prepared in Tris-EDTA buffer, absorbance at $405 \mathrm{~nm}$ was recorded, at $37^{\circ} \mathrm{C}$, for $20 \mathrm{~min}$ at $6 \mathrm{~s}$ intervals using a Versamax Microplate Reader.

Chromogenic assay for measuring factor Xa activity - The ability of SGL in interfering with factor Xa catalytic activity was evaluated using a chromogenic assay. FXa (1 nM, final concentration) was incubated for $10 \mathrm{~min}$ at RT in the presence of SGL serially diluted in HEPES-BSA buffer. After addition of $50 \mu \mathrm{L}$ of $200 \mu \mathrm{M}$ S-2765, absorbance at $405 \mathrm{~nm}$ was recorded at $37^{\circ} \mathrm{C}$ for 20 min at $6 \mathrm{~s}$ intervals in a Versamax Microplate Reader. Controls were performed in the absence of SGL.

Statistical analysis - Data were analysed by Student $t$ test comparing two groups. The analysis was performed using Prism 3.0 (GraphPad Software). In all cases, results were considered to be significantly different for $\mathrm{p}<0.05$.

\section{RESULTS}

L. amazonesis metacyclic promastigotes activate blood coagulation in a PS-dependent manner - A number of studies have reported presence of PS on Leishmania parasites (Wassef et al. 1985, Henriques et al. 2003, Wanderley et al. 2006, Yoneyama et al. 2006, França-Costa et al. 2012, Farias et al. 2013). However this phenomenon has been refuted by other groups that were unable to detect this phospholipid on viable parasites (Zufferey et al. 2003, Zhang et al. 2007, Zheng et al. 2010, Weingärtner et al. 2012). In this study we demonstrate that metacyclic promastigote forms of L. amazonensis expose PS in vi- able parasites. Fig. 1A shows a parasite population that stains for annexin $\mathrm{V}$, but is negative for labelling with PI, a known marker of cell membrane rupture. PS exposure by Leishmania amastigote forms accelerate blood coagulation in vitro, as previously demonstrated in parasites isolated from BALB/c mice lesions (Balanco et al. 2001). In this study we expand this observation to in vitro generated metacyclic promastigotes. Fig. 1B shows that incubation of human plasma with $10^{5}$ and $10^{6}$ parasites reduced the human plasma coagulation time by $32 \%$ and $28 \%$, respectively, as compared to control, thus evidencing a parasite-number dependent procoagulant effect.

Thrombin is the last enzyme generated in the clotting cascade. It is generated by the cleavage of prothrombin by prothrombinase complex, which consists in a calciumdependent, membrane-assembled complex between the enzyme factor Xa and its cofactor factor Va. Assembly of the prothrombinase complex on PS-containing mem-
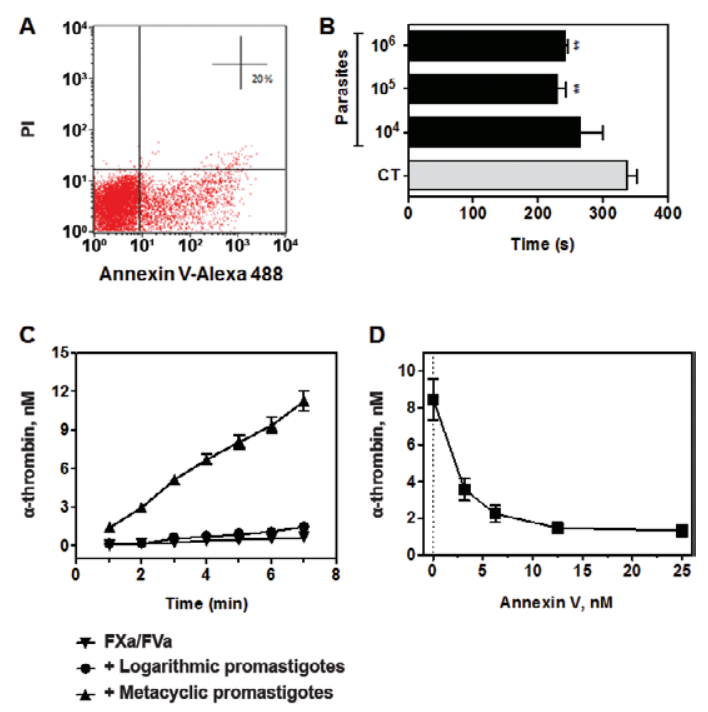

Fig. 1: procoagulant activity of Leishmania amazonensis metacyclic promastigotes. A: analysis of phosphatidylserine exposure on metacyclic promastigotes. Parasites were analysed by flow cytometry in a dot plot of Alexa 488 conjugated-annexin V vs. propidium iodide (PI) double staining. Quadrant regions were defined from a negative control with no labelling. Data are representative of two independent experiments; B: recalcification time of human plasma was measured in the absence (CT) or in the presence of different numbers of metacyclic promastigotes. Results from 10 independent experiments are expressed as mean \pm standard error of the means (SEM). Asterisks mean $\mathrm{p}<0.01$ relative to $\mathrm{CT}$; $\mathrm{C}:$ L. amazonensis metacyclic promastigotes support the prothrombinase complex assembly. Activation of prothrombin into thrombin by factor Xa/factor Va was performed in the absence or in the presence of logarithmic or metacyclic promastigotes. The amount of thrombin formed was quantified as described in the Materials and Methods section. Data from three independent experiments are expressed as mean \pm SEM; D: annexin V blocks the assembly of the prothrombinase complex on L. amazonensis. Metacyclic promastigotes were pre-incubated with different concentrations of annexin $\mathrm{V}$ and further assayed for thrombin generation in the presence of factor Xa/factor Va as described in the Materials and Methods section. Data from three independent experiments are expressed as mean \pm SEM. Data were analysed by Student $t$ test. 
branes is essential to achieve a high catalytic efficiency (Mann 1999). In order to confirm PS exposure in L. amazonensis, we further evaluated the ability of parasites in promoting thrombin formation by the prothrombinase complex. Fig. 1C shows that metacyclic promastigotes, but not logarithmic promastigotes, support the coagulation complex assembly resulting in prothrombin activation into thrombin. This process was highly dependent on PS exposure, since pre-incubation of parasites with annexin V completely blocked thrombin formation (Fig. 1D).

Lu. longipalpis saliva counteracts L. amazonensis procoagulant activity - A number of anticoagulant proteins have been identified in $L u$. longipalpis saliva (Charlab et al. 1999, Valenzuela et al. 2004, Collin et al. 2012). In accordance with these studies, the presence of one SGL caused a 2.5-fold increase in plasma coagulation time, as compared to control in the absence of SGL (Fig. 2A). Next, we investigated the putative coagulation pathways inhibited by SGL by means of the activated partial aPTT and PT tests. Our data showed that the coagulation time was not altered in the presence or in the absence of one SGL as evaluated by the PT test (Fig. $2 \mathrm{~A}$ ), which contains tissue factor and is used to screen the extrinsic pathway. On the other hand, the aPTT test, which is used to evaluate the intrinsic pathway, showed an increase of $25 \%$ in the coagulation time when one SGL was added to the reaction medium (Fig. 2A).

Considering that the procoagulant activity of L. amazonensis metacyclic forms might be deleterious to vector blood feeding (Kimblin et al. 2008, Secundino et al. 2012), we next analysed the counteracting effect of $L u$. longipalpis saliva on L. amazonensis procoagulant activity. Fig. 2B shows that $0.5 \mathrm{SGL}$ efficiently counteracted the procoagulant effect of either $10^{5}$ or $10^{6}$ parasites. Alternatively, assays performed at a fixed concentration of $10^{6}$ parasites and varying SGL amounts showed a dosedependent inhibitory effect of sandfly saliva (Fig. 2C).

Sandfly saliva inhibits prothrombinase complex activity - Plasma coagulation results from the enzymatic cleavage of soluble fibrinogen into an insoluble fibrin polymer. This reaction is specifically catalysed by thrombin in a reaction that is subsequent to massive prothrombin activation by membrane-assembled prothrombinase complex (Mann 1999). In this context, we further evaluated the ability of Lu. longipalpis saliva in inhibiting the prothrombinase complex. This was assessed by incubating saliva with factor Xa, factor Va and prothrombin in the presence of calcium ions and artificial PC/PS membranes. As shown in Fig. 3A, the presence of increasing SGL concentrations progressively inhibited thrombin formation by PC/PS-assembled prothrombinase complex. We next examined the ability of sandfly saliva to inhibit $L$. amazonensis-assembled prothrombinase complex. Fig. 3B shows that the presence of one SGL efficiently counteracted thrombin formation in all parasite concentrations tested.

Sandfly saliva inhibits tenase complex and FXa activity - Activation of zymogen factor $\mathrm{X}$ into factor $\mathrm{Xa}$ is efficiently catalysed by the intrinsic tenase complex, which consists in a calcium-dependent, membrane-assembled complex between the enzyme factor IXa and its cofactor factor VIIIa (Mann 1999). In order to evaluate whether the intrinsic tenase complex is inhibited by sandfly saliva, we incubated factor IXa, factor VIIIa and $\mathrm{PC} / \mathrm{PS}$ vesicles with serially diluted sandfly saliva. Fig. 4A shows that increasing SGL concentrations progressively decrease factor $\mathrm{X}$ activation.

In general, many arthropods anticoagulants target thrombin or factor $\mathrm{X}$ and/or factor Xa, this last a nexus of the intrinsic and extrinsic pathways of blood coagulation (Champagne 2005). In order to evaluate whether the sandfly saliva anticoagulants have a direct effect on factor Xa and can thus contribute to extend the observed coagulation time, chromogenic assays were performed with factor Xa and sandfly saliva. As shown in Fig. 4B, the presence of 0.5 SGL caused a $50 \%$ inhibition in fac-
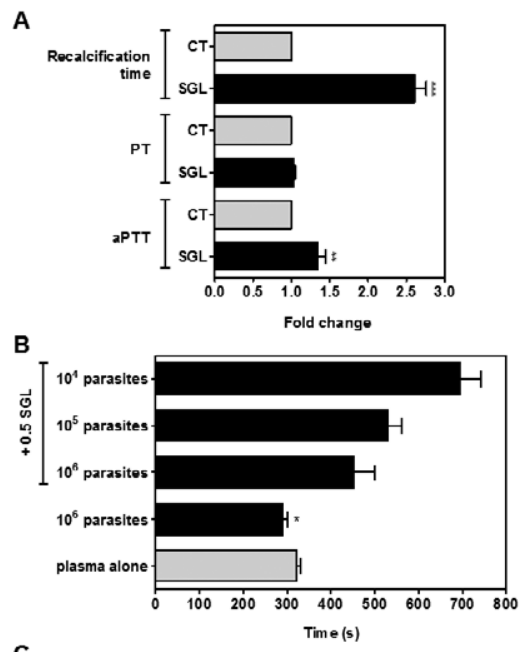

C

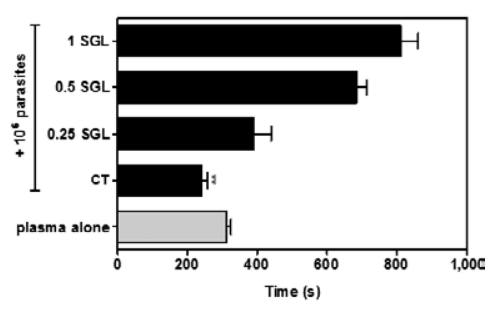

Fig. 2: anticoagulant effect of Lutzomyia longipalpis saliva counteracts procoagulant activity of Leishmania amazonensis metacyclic promastigotes. A: anticoagulant activity of sandfly saliva. Recalcification time of human plasma was measured in the absence (CT) or in the presence of salivary gland lysate (SGL). Prothrombin time and thromboplastin time assays were also performed in the absence (CT) or in the presence of SGL. In all cases, one SGL per assay was used. Data from three independent experiments are expressed as mean \pm standard error of the means (SEM). Asterisks mean $p<0.001$ or $p<0.01$ relative to CT; B: recalcification time of human plasma was measured in the absence (plasma alone) or in the presence of different numbers of promastigotes, co-incubated or not with $0.5 \mathrm{SGL}$; C: recalcification time of human plasma was measured in the absence (plasma alone) or in the presence of $10^{6}$ promastigotes, co-incubated or not with different numbers of SGL. In B and C, results from 10 independent experiments are expressed as mean \pm SEM. Data were analysed by Student $t$ test. *: $\mathrm{p}<0.05 ; * * \mathrm{p}<0.01$ (relative to control). 
tor Xa catalytic activity, thus indicating that the anticoagulant effect of sandfly saliva is, at least in part, related to direct inhibition of factor Xa activity.

\section{DISCUSSION}

The loss of membrane asymmetry during programmed cell death, i.e. apoptosis, is typically associated with PS exposure. The presence of PS in Leishmania parasites has been reported by a number of groups (Wassef et al. 1985, Henriques et al. 2003, Wanderley et al. 2006, 2009, Yoneyama et al. 2006, França-Costa et al. 2012, Farias et al. 2013) and the phenomenon of PS exposure by viable parasites has been named "apoptotic mimicry". This process is often referred to as a strategy of the parasites to evade host inflammatory and immune responses (Wanderley et al. 2006, Mercer \& Heleniu 2008, Laliberte \& Moss 2009, Santos et al. 2011). This process, which resembles PS exposure during apoptosis, consists in the externalisation of PS on living parasite's surface. In the case of Leishmania it is believed that most of the amastigote forms expose PS without evidences of apoptotic death (Balanco et al. 2001, Wanderley et al. 2006). Interestingly, it has been reported a differential PS exposure between amastigotes isolated from either susceptible or resistant mice (Wanderley et al. 2006). On the other hand, in promastigotes, a subpopulation of metacyclic forms exposes PS due to apoptosis. The recognition of PS-exposing metacyclic promastigotes

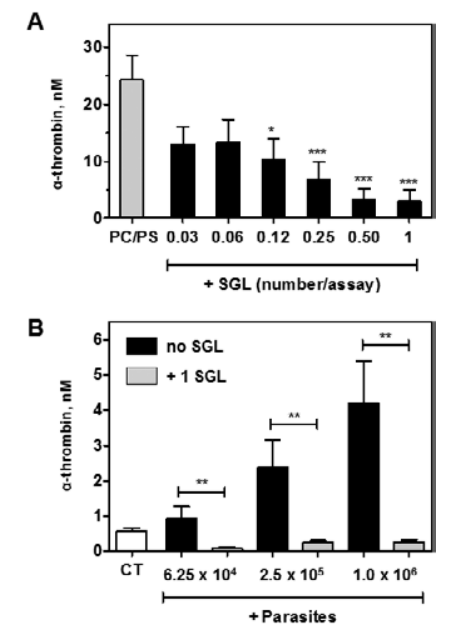

Fig. 3: inhibition of prothrombinase complex by Lutzomyia longipalpis saliva. A: activation of prothrombin into thrombin was carried out in the presence of factor Xa, factor Va and L- $\alpha$-phosphatidylcholine (PC)/phosphatidylserine (PS) vesicles as described in the Materials and Methods section. Assays were performed in the presence of serially diluted salivary gland lysate (SGL) or HEPES-bovine serum albumin buffer alone (PC/PS). Data from five independent experiments are expressed as mean \pm standard error of the means (SEM) [***: $\mathrm{p}<0.001 ; *: \mathrm{p}<0.05$ (relative to control) (PC/PS)]; B: inhibition of prothrombinase complex by SGL ( 0.5 equivalent per assay) in the presence of different numbers of Leishmania amazonensis metacyclic promastigotes. A negative control of thrombin generation in the presence of factor $\mathrm{Xa} /$ factor $\mathrm{Va}$ alone and in the absence of parasites and SGL was also included (CT). Results from three independent experiments are expressed as mean \pm SEM. Data were analysed by Student $t$ test. ${ }^{* *}: \mathrm{p}<0.01$. by phagocytic cells modulates host immune response by eliciting the production of transforming growth factorbeta and interleukin-10, which facilitate the establishment and maintenance of the infection by the non-PS exposing metacyclic forms (van Zandbergen et al. 2006, Wanderley et al. 2009). In this study we demonstrate that metacyclic promastigote forms of L. amazonensis expose PS, while not presenting evidences of cell membrane rupture.

PS exposure by activated platelets is an essential phenomenon during physiological haemostasis (Heemskerk et al. 2002, Lentz 2003). In fact, PS-containing membranes allow the assembly of multimolecular enzymatic complexes that ultimately lead to thrombin generation and fibrin deposition (Mann 1999). As a result of PS exposure, we herein demonstrate that L. amazonensis accelerates plasma clotting and supports the assembly of the prothrombin activating complex, prothrombinase. This process was fully dependent of PS exposure since annexin $\mathrm{V}$ completely reverted thrombin formation in the presence of L. amazonensis. The ability of L. amazonensis in recruiting and activating coagulation proteins might be correlated with non-haemostatic properties of these proteins. In this context it has been demonstrated that activation of protease-activated receptor-1, known as the thrombin receptor, contribute to L. amazonensis infection in macrophages (Rana et al. 2011).

Saliva of haematophagous arthropods contains a diverse mixture of compounds that counteracts host haemostasis (Ribeiro \& Francischetti 2003). These com-
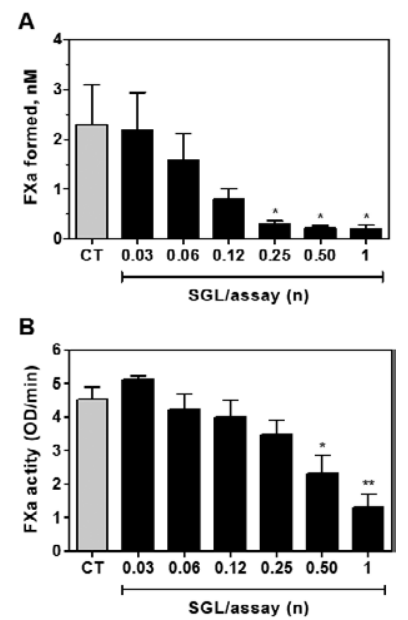

Fig. 4: inhibition of intrinsic tenase complex and factor Xa catalytic activity by Lutzomyia longipalpis saliva. A: activation of factor $\mathrm{X}$ was carried out in the presence of factor VIIIa, factor IXa and L- $\alpha$ phosphatidylcholine/phosphatidylserine vesicles as described in the Materials and Methods section. Assays were performed in the presence of serially diluted salivary gland lysate (SGL) or HEPES-bovine serum albumin (BSA) buffer alone (CT). Data from three independent experiments are expressed as mean \pm standard error of the means (SEM) (asterisk means $\mathrm{p}<0.05$ relative to $\mathrm{CT}$ ); B: factor Xa catalytic activity was assayed by measuring the cleavage of S-2765 chromogenic substrate in the absence (CT) or in the presence of different numbers of SGL serially diluted in HEPES-BSA buffer. Data from three independent experiments are expressed as mean \pm SEM. Data were analysed by Student $t$ test. **: $\mathrm{p}<0.01 ;{ }^{*}: \mathrm{p}<0.05$ (relative to CT). 
pounds are essential for achieving a successful blood feeding. In this context, Charlab et al. (1999) have shown that salivary glands from $\mathrm{Lu}$. longipalpis displays potent anticoagulant effect. An anti-clotting molecule containing a carbohydrate-recognition domain-like structure, with a C-type $\left(\mathrm{Ca}^{+2}\right.$-dependent) lectin domain, similar to the snake venom active proteins has been identified (Kini 2006). Six other members of a family of putative anticoagulants were identified on secreted proteins from Lu. longipalpis saliva (Valenzuela et al. 2004). Our results confirmed the anticoagulant effect of Lu. longipalpis saliva. A prolonged aPTT indicated a specific effect in the intrinsic coagulation pathway. However, the effect of saliva towards extrinsic pathway can be eventually underestimated, due to the large amount of thromboplastin reagent that is used in the PT test. This observation agrees with the estimated concentration of the factor Xa inhibitor, lufaxin, which has been recently characterised in the salivary gland of Lu. longipalpis (Collin et al. 2012). Indeed, our results demonstrate that the anticoagulant effect of sandfly saliva is partially due to the direct inhibition of factor Xa activity, resulting in decreased thrombin formation by prothrombinase complex and decreased factor Xa activity towards a chromogenic substrate. In this context, lufaxin specifically inhibits factor $\mathrm{Xa}$ in a tight, reversible, $\mathrm{Ca}^{+2}$-independent and non-competitive manner (Collin et al. 2012). Remarkably, sandfly saliva counteracts $L$. amazonensis procoagulant activity as well as thrombin formation by L. amazonensisassembled prothrombinase complex.

It is important to emphasise that other anticoagulant proteins might exist in the saliva of Lu. longipalpis. While anophelines display thrombin-directed anticoagulants, culicine mosquitoes display factor Xa-directed anticoagulants (Stark \& James 1996, Pérez de León et al. 1998). In Anopheles albimanus, anophelin, a $6.5 \mathrm{kDa}$ salivaderived peptide, functions as a specific and tight-binding thrombin inhibitor (Francischetti et al. 1999). In addition, a Kazal-type thrombin inhibitor has been also characterised in Aedes aegypti (Watanabe et al. 2011). In the mosquito vector of yellow fever, Aedes albopictus, the protein alboserpin, a factor Xa inhibitor, was characterised as an atypical serpin, which binds tightly to factor $\mathrm{Xa}$ and also contains high affinity for heparin and interacts with PC and phosphatidyl ethanolamine (Calvo et al. 2011).

Altogether we conclude that the previously described immunosuppressive properties evoked by PS exposure on L. amazonensis parasites are concomitant with procoagulant responses. On the other hand, the potent anticoagulant effect of Leishmania parasites' vector's saliva counteracts this effect. Most of the anticoagulant properties of haematophagous organisms are driven against host procoagulant enzymes or multimolecular coagulation complexes. Therefore it is conceivable that vector's saliva impairs procoagulant, but not immunosuppressive properties of PS exposing parasites in the first steps of infection. On the other hand, the subsequent spread of the parasite on the vertebrate host possibly unmasks the procoagulant properties of PS exposing parasites which may evoke important biological responses during Leishmania infection.

\section{REFERENCES}

Astermark J, Hogg PJ, Bjork I, Stenflo J 1992. Effects of gammacarboxyglutamic acid and epidermal growth fator-like modules of factor IX on factor X activation. Studies using proteolytic fragments of bovine factor IX. $J$ Biol Chem 267: 3249-3256.

Balanco JMF, Moreira ME, Bonomo A, Bozza PT, Amarante-Mendes G, Pirmez C, Barcinski M 2001. Apoptotic mimicry by an obligate intracellular parasite downregulates macrophage microbicidal activity. Curr Biol 11: 1870-1873.

Calvo E, Mizurini DM, Sá-Nunes A, Ribeiro JM, Andersen JF, Mans BJ, Monteiro RQ, Kotsyfakis M, Francischetti IM 2011. Alboserpin, a factor Xa inhibitor from the mosquito vector of yellow fever, binds heparin and membrane phospholipids and exhibits antithrombotic activity. J Biol Chem 286: 27998-28010.

Champagne DE 2005. Antihemostatic molecules from saliva of bloodfeeding arthropods. Pathophysiol Haemost Thromb 34: 221-227.

Charlab R, Valenzuela JG, Rowton ED, Ribeiro JM 1999. Toward an understanding of the biochemical and pharmacological complexity of the saliva of haematophagous sandfly Lutzomyia longipalpis. Proc Natl Acad Sci USA 96: 15155-15160.

Collin N, Assumpção TC, Mizurini DM, Gilmore DC, Dutra-Oliveira A, Kotsyfakis M, Sá-Nunes A, Teixeira C, Ribeiro JM, Monteiro RQ, Valenzuela JG, Francischetti IM 2012. Lufaxin, a novel factor Xa inhibitor from the salivary gland of the sandfly Lutzomyia longipalpis blocks protease-activated receptor 2 activation and inhibits inflammation and thrombosis in vivo. Arterioscler Thromb Vasc Biol 32: 2185-2198.

Farias LHS, Rodrigues APD, Silveira FT, Seabra SH, DaMatta RA, Saraiva EM, Silva EO 2013. Phosphatidylserine exposure and surface sugars in two Leishmania (Viannia) braziliensis strains involved in cutaneous and mucocutaneous leishmaniasis. J Infect Dis 207: 537-543.

Fernandes RS, Kirszberg C, Rumjanek VM, Monteiro RQ 2006. On the molecular mechanisms for the highly procoagulant pattern of C6 glioma cells. J Thromb Haemost 4: 1546-1552.

França-Costa J, Wanderley JL, Deolindo P, Zarattini JB, Costa J, Soong L, Barcinski MA, Barral A, Borges VM 2012. Exposure of phosphatidylserine on Leishmania amazonensis isolates is associated with diffuse cutaneous leishmaniasis and parasite infectivity. PLOS ONE 7: e36595.

Francischetti IM, Seydel KB, Monteiro RQ, Whitten RO, Erexson CR, Noronha AL, Ostera GR, Kamiza SB, Molyneux ME, Ward JM, Taylor TE 2007. Plasmodium falciparum-infected erythrocytes induce tissue factor expression in endothelial cells and support the assembly of multimolecular coagulation complexes. $J$ Thromb Haemost 5: 155-165.

Francischetti IM, Valenzuela JG, Ribeiro JM 1999. Anophelin: kinetics and mechanism of thrombin inhibition. Biochemistry 38 : 16678-16685.

Heemskerk JW, Bevers EM, Lindhout T 2002. Platelet activation and blood coagulation. Thromb Haemost 88: 186-193.

Henriques C, Atella GC, Bonilha VL, de Souza W 2003. Biochemical analysis of proteins and lipids found in parasitophorous vacuoles containing Leishmania amazonensis. Parasitol Res 89: 123-133.

Kimblin N, Peters N, Debrabant A, Secundino N, Egen J, Lawyer P, Fay MP, Kamhawi S, Sacks D 2008. Quantification of the infectious dose of Leishmania major transmitted to the skin by single sandflies. Proc Natl Acad Sci USA 105: 10125-10130.

Kini RM 2006. Anticoagulant proteins from snake venoms: structure, function and mechanism. Biochem J 397: 377-387. 
Kirszberg C, Lima LG, de Oliveira AS, Pickering W, Gray E, Barrowcliffe TW, Rumjanek VM, Monteiro RQ 2009. Simultaneous tissue factor expression and phosphatidylserine exposure account for the highly procoagulant pattern of melanoma cell lines. Melanoma Res 19: 301-308.

Koh CY, Kini RM 2009. Molecular diversity of anticoagulants from haematophagous animals. Thromb Haemost 102: 437-453.

Laliberte JP, Moss B 2009. Appraising the apoptotic mimicry model and the role of phospholipids for poxvirus entry. Proc Natl Acad Sci USA 106: 17517-17521.

Lentz BR 2003. Exposure of platelet membrane phosphatidylserine regulates blood coagulation. Prog Lipid Res 42: 423-438.

Lima LG, Oliveira AS, Campos LC, Bonamino M, Chammas R, Werneck C, Vicente CP, Barcinski MA, Petersen LC, Monteiro RQ 2011. Malignant transformation in melanocytes is associated with increased production of procoagulant microvesicles. Thromb Haemost 106: 712-723.

Mann KG 1999. Biochemistry and physiology of blood coagulation. Thromb Haemost 82: 165-174.

Mercer J, Helenius A 2008. Vaccinia virus uses macropinocytosis and apoptotic mimicry to enter host cells. Science 320: 531-535.

Monteiro RQ 2005. Targeting exosites on blood coagulation proteases. An Acad Bras Cienc 77: 275-280.

Pérez de León AA, Valenzuela JG, Tabachnick WJ 1998. Anticoagulant activity in salivary glands of the insect vector Culicoides variipennis sonorensis by an inhibitor of factor Xa. Exp Parasitol 88: 121-130.

Rana T, Misra S, Mittal MK, Farrow AL, Wilson KT, Linton MF, Fazio S, Willis IM, Chaudhuri G 2011. Mechanism of down-regulation of RNA polymerase III-transcribed non-coding RNA genes in macrophages by Leishmania. J Biol Chem 286: 6614-6626.

Ribeiro JM, Francischetti IM 2003. Role of arthropod saliva in blood feeding: sialome and post-sialome perspectives. Аnпu Rev Entomol 48: 73-88.

Santos TA, Portes JA, Damasceno-Sá JC, Caldas LA, Souza WD, DaMatta RA, Seabra SH 2011. Phosphatidylserine exposure by Toxoplasma gondii is fundamental to balance the immune response granting survival of the parasite and of the host. PLoS ONE 6: e27867.

Secundino NF, de Freitas VC, Monteiro CC, Pires AC, David BA, Pimenta PF 2012. The transmission of Leishmania infantum chagasi by the bite of the Lutzomyia longipalpis to two different vertebrates. Parasite Vectors 5: 20.

Späth GF, Beverley SM 2001. A lipophosphoglycan-independent method for isolation of infective Leishmania metacyclic promastigotes by density gradient centrifugation. Exp Parasitol 99: 97-103.

Stark KR, James AA 1996. Salivary gland anticoagulants in culicine and anopheline mosquitoes (Diptera: Culicidae). J Med Entomol 33: 645-650.
Valenzuela JG, Garfield M, Rowton ED, Pham VM 2004. Identification of the most abundant secreted proteins from the salivary glands of the sandfly Lutzomyia longipalpis, vector of Leishmania chagasi. J Exp Biol 207: 3717-3729.

van Zandbergen G, Bollinger A, Wenzel A, Kamhawi S, Voll R, Klinger M, Müller A, Hölsch C, Herrmann M, Sacks D, Solbach W, Laskay T 2006. Leishmania disease development depends on the presence of apoptotic promastigotes in the virulent inoculum. Proc Natl Acad Sci USA 103: 13837-13842.

Wanderley JL, Barcinski MA 2010. Apoptosis and apoptotic mimicry: the Leishmania connection. Cell Mol Life Sci 67: 1653-1659.

Wanderley JL, da Silva LHP, Deolindo P, Soong L, Borges VM, Prates DB, de Souza AP, Barral A, Balanco JM, do Nascimento MT, Saraiva EM, Barcinski MA 2009. Cooperation between apoptotic and viable metacyclics enhances the pathogenesis of leishmaniasis. PLoS ONE 4: e5733.

Wanderley JL, Moreira ME, Benjamin A, Bonomo AC, Barcinski MA 2006. Mimicry of apoptotic cells by exposing phosphatidylserine participates in the establishment of amastigotes of Leishmania (L.) amazonensis in mammalian hosts. J Immunol 176: 1834-1839.

Wassef MK, Fioretti TB, Dwyer DM 1985. Lipid analyses of isolated surface membranes of Leishmania donovani promastigotes. Lipids 20: 108-115.

Watanabe RM, Tanaka-Azevedo AM, Araujo MS, Juliano MA, Tanaka AS 2011. Characterization of thrombin inhibitory mechanism of rAaTI, a Kazal-type inhibitor from Aedes aegypti with anticoagulant activity. Biochimie 93: 18-23.

Weingärtner A, Kemmer G, Müller FD, Zampieri RA, dos Santos MG, Schiller J, Pomorski TG 2012. Leishmania promastigotes lack phosphatidylserine, but bind annexin $\mathrm{V}$ upon permeabilization or miltefosine treatment. PLoS ONE 7: e42070.

Yoneyama KA, Tanaka AK, Silveira TG, Takahasha HK, Straus AH 2006. Characterization of Leishmania (Viannia) braziliensis membrane microdomains and their role in macrophage infectivity. J Lipid Res 47: 2171-2178.

Zhang K, Pompey JM, Hsu FF, Key P, Bandhuvula P, Saba JD, Turk J, Beverley SM 2007. Redirection of sphingolipid metabolism toward de novo synthesis of ethanolamine in Leishmania. EMBO J 26: $1094-1104$

Zheng L, T'Kind R, Decuypere S, von Freyend SJ, Coombs GH, Watson DG 2010. Profiling of lipids in Leishmania donovani using hydrophilic interaction chromatography in combination with Fourier transform mass spectrometry. Rapid Commun Mass Spectrom 24: 2074-2082.

Zufferey R, Allen S, Barron T, Sullivan DR, Denny PW, Almeida C, Smith DF, Turco SJ, Ferguson MA, Beverley SM 2003. Ether phospholipids and glycosylinositol phospholipids are not required for amastigote virulence or for inhibition of macrophage activation by Leishmania major. J Biol Chem 278: 44708-44718. 